\title{
Tooth polishing - A mouthful of history
}

\author{
Avineet Kaur ${ }^{1}$, Monika Gupta ${ }^{2, *}$, Debdutta Das ${ }^{3}$, Surinder Sachdeva ${ }^{4}$, Sanjeev Jain ${ }^{5}$ \\ ${ }^{1}$ Senior Lecturer, ${ }^{2}$ Professor, ${ }^{3-5}$ Professor \& HOD, ${ }^{1,4,5}$ Dept. of Periodontics, ${ }^{2,3}$ Dept. of Oral Surgery, ${ }^{1-4} \mathrm{M}$ M College of Dental \\ Sciences and Research Mullana, Haryana, ${ }^{5}$ Guru Nanak Dev Dental College \& Research Institute, Patiala, Punjab, India
}

*Corresponding Author:

Email: monikabox@yahoo.com

\begin{abstract}
Tooth polishing continues to be an integral part of clinical practice since the concept of selective polishing was introduced in the 1980s. Polishing essenitiates the removal of stains and plaque bio-film and provides a method for applying various medicaments to the teeth such as desensitizing agents. With passage of time emergence of more efficient and effective devices like jet abrasives have been introduced. Still the role of rubber cups with prophy angles cannot be overlooked as they are still being widely used and provide an economical alternative.

A traditional polishing method, i.e. a rubber-cup with prophylaxis paste, has been shown to remove the fluoride-rich outer layer of the enamel thus causing significant loss of cementum and dentin over time. So alternative tooth polishing methods were developed with evidence to support alternative tooth polishing methods, dentists should familiarize themselves with contemporary methods including air polishing. The purpose of this review is to provide a comprehensive overview of recent advancements in air polishing.

Clinical Relevance

Scientific Rationale of Polishing: Polishing cause the abrasion of tooth surfaces. American dental hygienists association strongly recommends, do polishing of the tooth surfaces, when a dental hygienist or dentist determines a specific need for it. One should understand patient's expectations they simply like the look and feel of teeth along with the taste and smell are next important factors.

Principal findings: Polishing has been evolved from finely ground coral, eggs, shells, ginger or salt. After that various other polishing powders like flour of pumice and calcium carbonate, aluminum oxide (alumina), silicon carbide, aluminum silicate, silicon dioxide, carbide compounds, garnet, feldspar, zirconium silicate, zirconium oxide, and boron. Because of the particle size they were considered to be more abrasive.

Practical Implications: With new advances finer abrasive agents with fine particle sizes have been invented, which causes less of abrasion and provide a good sweet taste. Various powders like Erythritol and Glycine with finer particle size have been developed for polishing to achieve smooth shining surfaces.
\end{abstract}

Keywords: Polishing, Stains, Glycine, Prophylaxis agents.

\section{Introduction}

Periodontal diseases are multi-factorial in nature and are caused by microorganisms that colonize the tooth surface at or below the gingival margin. ${ }^{1}$ Its treatment necessitates thorough supra- and subgingival calculus and biofilm removal which is also the central part of the periodontal maintenance therapy. For debridement purposes, hand instruments, sonic or ultrasonic scalars may be used ${ }^{2}$. Even if instrumentation is performed by well trained and proficient clinicians, the use of these instruments periodically may render surfaces of the teeth rough apart from leaving stains on them. This gives rise to the need for polishing the tooth surfaces, which can be accomplished by the conventional rubber cup prophylaxis and air polishing. ${ }^{2}$

The conventional rubber cup prophylaxis and the air powder polishing system are both effective professional techniques for plaque and stain removal. ${ }^{3}$ For over half a century, the use of rubber cup and paste has been the most common method of prophylaxis. ${ }^{4}$

Various abrasive powders like sodium bicarbonate (treated with up to $0.8 \%$ of Silicium oxide or Tricalcium phosphate to enhance hydrophobicity in order to sustain powder flow characteristics),
Aluminium Trihydroxide, Sodium phosphosilicate (bioactive glass), Calcium carbonate and Glycine air polishing powder are available to be used with air polishing devices. ${ }^{5}$

\section{Historical perspective}

During historic era Porte polishers were used for removing extrinsic stains or application of treatment agents such as for hypersensitive areas. It uses wooden points. The wooden points used are various kinds of wood preferably orangewood. ${ }^{6}$ Main drawbacks of this procedure are slowness and amount of hand strength for instrumentation. Finishing or polishing strips are used for inter-proximal areas or line angles but should be cautiously used to avoid cutting of soft tissues as these are highly abrasive. During half a century ago, the most common method of tooth polishing is using rubber cup and pumice, and the widely used polishing paste consists of flour of pumice, glycerin, colour additive and in addition, sodium fluoride $(\mathrm{NaF})$ or stannous fluoride (SnF) might be added to this mix for desensitizing effect. ${ }^{4}$

Rationale behind polishing, using a pumice or an abrasive paste is placed on a rubber cup and applied to 
the clinical crowns of teeth using a rotating cup at slow speed. Protocol was first reported in the 1960s, with assumptions that materia alba, plaque and other natural coatings on tooth surfaces acts as a barrier to fluoride uptake, thus reducing the efficacy of topical fluoride application. So this logical step made by many clinicians, initiates the removal of the substances adherent to the enamel by professional prophylaxis will enhance the efficacy of professionally applied topical fluoride, and is also expected to reduce the incidence of caries. $^{7}$

\section{Cleansing and Polishing instruments}

Rubber Cups: It consists of rubber shell with or without webbed configurations in hollow interior. These are used in hand pieces with special prophylaxis angle. It should be sterilized after every patient or disposable rubber cup and prophylaxis angle may be used. Good cleansing paste containing fluoride must be used and kept moist to minimize frictional heat. Polishing pastes are available with different grits i.e. fine, medium and coarse. ${ }^{8}$

Rubber Polishing Points: These rubber points screws up into the prophylaxis angle. These are made up of natural rubber. It is flexible in nature so that tip adapts to proximal surfaces, embrasures and around orthodontic bands and brackets. As ribs hold prophylaxis paste on the external surface so reapplication is less frequently required. ${ }^{9}$

Dental Tape: Used with the help of polishing paste and used in areas which are inaccessible to other polishing methods i.e. proximal surfaces. The tape is passed interproximally with firm labio-lingual motion. One should be careful thus preventing injury to gingiva and should be cleaned with warm water to remove all remnants of paste.

Bristle Brush: It is available as wheel and cup shapes. The bristle is used in the prophylaxis angle with a polishing paste. As bristles are stiff so usage should be confined to the crown to avoid injury of the cementum and the gingiva. ${ }^{8}$

Air Powder Polishing: The first air polisher handpiece deliver air-powdered slurry was used in the early 1980's. This device Prophy Jet is very effective in removing extrinsic stains and soft deposits. The slurry removes stains rapidly and efficiently by mechanical abrasion and provides warm water for rinsing and lavage. Air powder polishing can be used safely on titanium implant surfaces. Patients with infectious diseases should not be treated with this device because of the large quantity of aerosol created. ${ }^{8}$

\section{Significance of polishing}

Polishing is used to smoothen the tooth surfaces so that surfaces are glossy and lustrous. Polishing is used to remove soft deposits and stains from tooth surface. During polishing plaque, biofilm, stains and acquired pellicle are removed. ${ }^{10}$

\section{Different abrasive agents in dentistry}

Abrasive agent is used to clean and smoothen the tooth surfaces. Abrasive agents present in the polishing paste are same as those in dentifrices. Difference being the particle size of the abrasive, which is more in professional prophylaxis pastes. Prophylaxis polishing pastes available are in combination of a binder, humectants, coloring agent, preservative, and flavoring agent. ${ }^{11}$ Various particle sizes are available ranging from coarse, medium to fine. Hard and rough-shaped particle size compounds produce more abrasive action than particles that are soft andsmooth. ${ }^{10}$

The most commonly used abrasives in polishing pastes are flour of pumice and calcium carbonate. Other abrasive particles used in commercial prophylaxis polishing pastes include aluminum oxide (alumina), silicon carbide, aluminum silicate, silicon dioxide, carbide compounds, garnet, feldspar, zirconium silicate, zirconium oxide, boron, and calcium carbonate. Others include the emery, silica, and perlite. ${ }^{10}$

\section{Air-polisher}

Air polishers have overcome conventional rubber cup polishing paste systems for supragingival plaque removal as it reaches surfaces which are inaccessible to a rotary device. ${ }^{10}$ Various abrasives used in air polishers are aluminum trihydroxide, calcium sodium phosphosilicate, calciumcarbonate and glycine. ${ }^{10}$

\section{Different powders used as abrasive agents}

Sodium bicarbonate-based powder $\left(\mathrm{NaHCO}_{3}\right)$ was the first powder to be used in air polishing. $\mathrm{NaHCO}_{3}$ powders are processed to form a powder with a particle size of up to $250 \mu \mathrm{m}$. However patients with conditions affected by sodium intake air polishing with $\mathrm{NaHCO}_{3}$ based powders is contraindicated. This is a concern for use of $\mathrm{NaHCO}_{3}$ on many patients including those with sodium-restricted diets and hypertension.

Recent developments include various powders glycine, calcium sodium phosphosilicate, calcium carbonate and aluminum trihydroxide. Glycine, calcium sodium phosphosilicate and calcium carbonate are less abrasive than traditional sodium bicarbonate-based powders. $^{12}$

Calcium sodium phosphosilicate powder, (CaNaO6P-Si) is a bioactive glass developed specifically air polishing procedures. It is a chemical compound of naturally occurring elements including calcium, phosphorus, silica and sodium.

Calcium carbonate $\left(\mathrm{CaCO}_{3}\right)$ consists of spherically agglomerated crystals. Usage of this mass of uniformly shaped round crystals will minimize surface abrasion as comparing to the irregularly shaped particles. Particle size of the $\mathrm{CaCO}_{3}$ powder is $45 \mu \mathrm{m}$ which is less than $\mathrm{NaHCO}_{3}$.

Aluminum trihydroxide $\left(\mathbf{A l}(\mathbf{O H})_{3}\right)$ is an alternative air polishing powder for patients on sodium restricted 
diets. Its particles are harder and comparable in size to sodium bicarbonate.

Various Inorganic salts have also been investigated and used as air polishing agents widely. ${ }^{12}$

Glycine (greek: sweet) is a nonessential amino acid and an important component of most polypeptides. Substrate for the endogenous synthesis of physiologically important substances is such as tetrahydrofolic acid, purine, heme, creatine and glutathione. Glycine powder is commercially available and it is produced by milling glycine crystals in an agate disc grinder. A powder mixture with a mean particle size of less than $45 \mu \mathrm{m}$ and a maximum particle size of $60 \mu \mathrm{m}$ is obtained. Glycine powder particle size is approximately four times smaller than sodium bicarbonate powder. ${ }^{13}$

Erythritol powder: Recently, a new abrasive erythritol powder (EPAP) with very low abrasiveness as comparable to glycine was introduced for subgingival air polishing. Erythritol is non-toxic in nature and chemically neutral. It is a water soluble polyol used widely as an artificial sweetener and as a food additive. Its promising chemical characteristics allow its binding of antiseptic substances, thus suggesting it to be suitable for subgingival biofilm removal. Recently it has been demonstrated that erythritol has an inhibitory effect to some periodontopathogenic bacteria such as Porphyromonas gingivalis. ${ }^{14}$

\section{Advantages and disadvantages of various methods}

Porte polisher: Hand-held device with an orange-wood point uses abrasive agent to polish tooth surfaces.

\section{Advantages}

1. It is portable

2. Malpositioned tooth surfaces can be easily cleaned.

3. It generates minimal thermal heat.

4. Does not produce noise like rotary instruments so more comfortable to patient.

5. Doesn't produce microbial aerosols. ${ }^{15}$

\section{Disadvantages}

Its time consuming and hand strength for instrumentation takes a longer time for polishing. ${ }^{10}$

\section{Engine-driven polishers}

These are widely used for their efficiency and efficacy. Polishers are attached to the appropriate hand piece or prophy-angle, which are either straight or contra-angled shanks. A rubber cup or brush is attached to the prophy-angle. Hand-piece should always be used at a steady slow pace of $2500-3000 \mathrm{rpm} .{ }^{16}$

\section{Advantage}

They can be used easily as patient compliance and acceptance are high.

It was reported by Miller and Hodges that it took 10 min (3.4 s per tooth) to treat the entire mouth so time consumed is less. ${ }^{17}$

\section{Disadvantage}

It is contraindicated in patients having allergies to latex as rubber cups are made up of latex.

\section{Air-powder polisher}

These air polishers use slurry of distilled water and various powders. Air polisher hand-piece is attached directly to the air/water connector or as a separate unit or in combination with ultrasonic scalers. ${ }^{10}$

It has overcome conventional rubber cup polishing paste systems as it is more convenient for subgingival plaque removal and it reaches surfaces that are inaccessible to a rotary device. ${ }^{18}$

\section{Advantages}

1. Air polishing reduces the operator and patient fatigue.

2. Air polishing requires less time.

3. It removes plaque from inaccessible areas that are otherwise difficult for rotary instruments to reach like furcations, flutings and close root proximities. $^{18}$

\section{Disadvantages}

1. It cannot be used in patients with respiratory, renal or metabolic disease, infectious disease, children, diuretics or long term steroid therapy.

2. Aerosols generated by air-polishing may present an infection control hazard. Hence, a pre procedural rinse is always recommended along with aerosol reduction devices. ${ }^{18}$

\section{Comparison of rubber cup polishing and air- polishing methods}

Various studies revealed that the effectiveness of air-polishing to the rubber cup polishing for bacterial plaque and stain removal demonstrate that both methods are equally effective. ${ }^{19}$ Although both methods were reported to cause some gingival trauma. ${ }^{19}$ GarciaGodoy and Medlock have studied and reported that air polishers are more effective for plaque and stain removal in pits and fissures. ${ }^{10}$

\section{Vector-system}

Vector system uses a polishing fluid to polish teeth. Braun $e t$ al. suggested use of polishing fluid containing hydroxyapatite or an abrasive fluid containing silicon carbide with a resonating device that deflects vibrations towards the tooth surface, thereby minimizing the forces that remove tooth substance. ${ }^{10}$

\section{Evaluation of polished surfaces}

Teeth needs to be inspected thoroughly using a mouth mirror, intra-oral light, compressed air and disclosing solution. If any remnants are left that has to be removed by either re-instrumentation or re-polishing the surface. Finishing strips or dental tape assists in the removal of residual inter-proximal stains. ${ }^{15}$ 


\section{Effects on Enamel, Cementum and Dentin}

Literature reviewed effects of air polishing on enamel, cementum and dentin removal by $\mathrm{NaHCO}$ 3.Studies have found air polishing to be safe on enamel with no significant loss of enamel and less abrasive than rubber-cup polishing. Tada et al studied the effect of nozzle distance which was placed at $6 \mathrm{~mm}$ at an angle of 45 degree and concluded that this angle and distance produced the shallowest defect depths. ${ }^{20}$

\section{Effects on Restorative Materials, Sealants, Orthodontic Appliances and Implants}

Gutmann concluded that clinicians should follow manufacturer's recommendations when using air polishing on restorative materials. Giacomelli et al found that on nanohybrid composite resin with glycine powder producing smaller surface defects (1 to $2 \mu \mathrm{m}$ wide) than $\mathrm{NaHCO} 3$ (5 to $10 \mu \mathrm{m}$ wide). ${ }^{12} \mathrm{~A}$ recent study by was conducted on patients with periimplantitis and found glycine powder significantly reduced bleeding on probing 6 months after treatment as comparative to patients who were treated with mechanical debridement using curets and chlorhexidine. ${ }^{21}$

\section{Health Concerns and Safety}

Very rare conditions that can arise from aerosols during air polishing include air emphysema, subcutaneous facial emphysema and pneumoparotitis. Flemmig discussed his findings from Health Device Alerts between 1977 and 2001, suggesting a total of 9 air emphysema and 3 air embolism incidents related to the use of APDs. ${ }^{22}$

Gutmann suggested universal precautions should be taken, using high-volume evacuation instead of a saliva ejector and pre- procedural rinse before treatment to prevent any potential health risks. ${ }^{12}$ These protocols are still suggested today.

\section{Trends and Future Research}

Glycine-based powder may become the airpolishing powder of choice due to its low abrasiveness on gingival tissues, tooth structure, restorative materials and its potential to clean both supragingival and subgingival surfaces. Research certainly supports that patients are accepting this technology and prefer. ${ }^{23,24}$

\section{Conclusion}

Tooth polishing used to be a part of a dental cleaning appointment. The main objective of the polishing is to smoothen teeth so that plaque and bacteria do not stick to the tooth easily. Teeth are polished only if stains are present which can be removed by scaling. This means that during cleaning procedures either some, all or none of the teeth may be polished. New polishing powders, such as glycine, are less abrasive. Future research is needed to explore ways to reduce aerosol production, and thus improving safety for all restorative materials and all patients, regardless of their medical condition.

\section{Conflict of Interest: None}

Sources of support in the form of grants: Not Applicable

\section{References}

1. Lindhe Mombelli A. Antibiotics in Periodontal Therapy. $5^{\text {th }}$ ed. Jan Lindhe, Niklaus P. Lang, Thorkild Karring. Clinical Periodontology and Implant Dentistry:Blackwell Munksgaard;2008.p.882-897.

2. Petersilka G, Faggion Jr CM, Stratmann U, Gerss J, Ehmke B, Haberlein. Effect of glycine powder air polishing on the gingiva. J Clin Periodontol. 2008;35:324-332.

3. Ramaglia L, Sbordone L, Ciaglia R N, Barone A, Martina R .A clinical comparison of the efficacy and efficiency of two professional prophylaxis procedures in orthodontic patients. Eur J Orthod. 1999;21:423-428.

4. Madan C, Bains R, and Bains VK .Tooth polishing: Relevance in present day periodontal Practice. J Ind Soc Periodontol .2009;13(1):58-59.

5. Petersilka G J. Subgingival air-polishing in the treatment of periodontal biofilm infections. Periodontol 2000. 2011;55:124-142.

6. Wilkins E.M.Clinical practice of the dental hygienist: $11^{\text {th }}$ ed.Wolters Kluwerp 698.

7. Azarpazhooh A and Main P.A. Efficacy of dental prophylaxis (rubber cup) for the prevention of caries and gingivitis: a systematic review of literature. bdj.2009:899.

8. Carranza F, Newman M, Takei H and Klokkevold P .Carranza's Clinical Periodontology. $9^{\text {th }}$ ed. St. Louis, Mo: Elsevier Saunders.580-581.

9. Wilkins E.M.Clinical practice of the dental hygienist: $11^{\text {th }}$ ed.Wolters Kluwerp 700.

10. Sawai MA, Bhardwaj A, Jafri Z, Sultan N, Daing A Tooth polishing: The current status. J Indian Soc Periodontol 2015;19:375-80.

11. Wilkins E.M.Clinical practice of the dental hygienist: $11^{\text {th }}$ ed.Wolters Kluwerp 694.

12. Graumann S.J, Sensat M.L, Stoltenberg JL. Air Polishing: A Review of Current Literature. J of dent hygiene. 2013;87(4):173-180.

13. Petersilka G.J. Subgingival air-polishing in the treatment of periodontal biofilm infections. J Periodontol.2011; 55: 124-142.

14. Hagi T T , Hofmanner P, Eick S, Donnet M, Salvi E, Sculean A et al. The effects of erythritol air polishing powder on microbiologic and clinical outcomes during supportive periodontal therapy: Six month results of a randomized controlled clinical trial.Quintessence Int. 2015;46(1):31-41.

15. Francis B, Barnes CM. Cosmetic and therapeutic polishing. In: Daniel SJ, Harfst SA, Wilder R, editor. Mosby's Dental Hygiene: Concepts, Cases and Competencies. Missouri: Elsevier; 2008. p. 599-622.

16. Rethman J. Polishing angles, cups and pastes. Pract Hyg 1997;1:32-3. Retraction in: Madan C, Bains R, Bains VK. Tooth polishing: Relevance in present day periodontal practice. J Indian Soc Periodontol 2009;13:58-9.

17. Hodges K.Concepts in Nonsurgurgical Periodontal Therapy.New York: Delmar;1998.345-366.

18. Boyde A. Airpolishing effects on enamel,dentine,cement and bone.Br Dent J 1984:156:287-291.

19. Braun A, Krause F, Frentzen M, Jepsen S. Removal of root substance with the Vector-system compared with 
conventional debridement in vitro. J Clin Periodontol.2005;32:153-157.

20. Tada K, Wiroj S, Inatomi M, Sato S. The characterization of dentin defects produced by air polishing. Odontology. 2012;100(1):41-46.

21. Sahm N, Becker J, Santel T, Schwarz F. Non-surgical treatment of peri-implantitis using an air-abrasive device or mechanical debridement and local application of chlorhexidine: a prospective, randomized,controlled clinical study. J Clin Periodontal. 2011;38(9):872-878.

22. Flemmig TF, Hetzel M, Topoll H, Gerss J, Haeberlein I, Petersilka G. Subgingival debridement efficacy of glycine powder air polishing. J Periodontol.2007;78(6):10021010.

23. Wennström JL, Dahlén G, Ramberg P. Subgingival debridement of periodontal pockets by air polishing in comparison with ultrasonic instrumentation during maintenance therapy. J Clin Periodontal. 2011;38(9):820 827.

24. Banerjee A, Hajatdoost-Sani M, Farrell S, Thompson. A clinical evaluation and comparison of bioactive glass and sodium bicarbonate air-polishing powders. J Dent. 2010;38(6):475-479. 